Dina Mönch, Jana Koch, Annika Maaß, Nicole Janssen, Thomas Mürdter, Philipp Renner, Petra Fallier-Becker, Wiebke Solaß, Matthias Schwab, Marc-H. Dahlke, Hans J. Schlitt and Tobias Leibold*

\title{
A human ex vivo coculture model to investigate peritoneal metastasis and innovative treatment options
}

https://doi.org/10.1515/pp-2021-0128

Received April 1, 2021; accepted June 15, 2021;

published online July 27, 2021

\section{Abstract}

Objectives: Peritoneal metastasis (PM) is commonly observed in patients with colorectal cancer (CRC). The outcome of these patients is poor, with an average survival of only six months without therapy, which requires a better understanding of PM biology and new treatment strategies. Methods: We established and characterized a human ex vivo peritoneal model to investigate the mechanisms of peritoneal seeding and possible treatment options. For this, CRC cell lines and patient-derived tumor organoids were cultured together with human peritoneum to investigate the invasion of malignant cells and the effects of local chemotherapy.

Results: Fresh human peritoneum was cultured for up to three weeks in a stainless steel ring system, allowing for survival of all peritoneal structures. Peritoneal cell

*Corresponding author: Dr. med. Tobias Leibold, Department of General and Visceral Surgery, Robert-Bosch-Hospital Auerbachstr. 110, 70376 Stuttgart, Germany, Phone: + 49071181013416 , E-mail: tobias.leibold@rbk.de Dina Mönch, Jana Koch, Annika Maaß, Nicole Janssen and Thomas Mürdter, Dr. Margarete Fischer-Bosch Institute for Clinical Pharmacology, Stuttgart, Germany; and University of Tübingen, Tübingen, Germany. https://orcid.org/0000-0002-2437-0394 (D. Mönch)

Philipp Renner, Department of General and Visceral Surgery, RobertBosch-Hospital, Stuttgart, Germany; and University Medical Center Regensburg, Regensburg, Germany

Petra Fallier-Becker and Wiebke Solaß, Institute of Pathology, University of Tübingen, Tübingen, Germany

Matthias Schwab, Dr. Margarete Fischer-Bosch Institute for Clinical Pharmacology, Stuttgart, Germany; and Departments of Clinical Pharmacology, Pharmacy, and Biochemistry, University of Tübingen, Tübingen, Germany

Marc-H. Dahlke, Department of General and Visceral Surgery, RobertBosch-Hospital, Stuttgart, Germany

Hans J. Schlitt, University Medical Center Regensburg, Regensburg, Germany

Ә Open Access. (C) 2021 Dina Mönch et al., published by De Gruyter. (cc) BY International License. survival was documented by light microscopy and immunohistochemical staining. Further, immunohistological characterization of the tissue revealed CD3positive T-lymphocytes and vimentin-positive fibroblasts within the peritoneum. In addition, extrace llular matrix components (collagens, matrix metalloproteinases) were localized within the tissue. Coculture with CRC cell lines and patient-derived CRC organoids revealed that cancer cells grew on the peritoneum and migrated into the tissue. Coculture with CRC cells confirmed that hyperthermal treatment at $41^{\circ} \mathrm{C}$ for 90 min significantly enhanced the intracellular entry of doxorubicin. Moreover, treatment with mitomycin C under hyperthermic conditions significantly reduced the amount of cancer cells within the peritoneum.

Conclusions: This human ex vivo peritoneal model provides a stringent and clinically relevant platform for the investigation of PM and for further elucidation of possible treatment options.

Keywords: colorectal cancer; ex vivo model; HIPEC; peritoneal metastasis; peritoneum.

\section{Introduction}

In 2020, colorectal cancer (CRC) ranked among the top five leading cancer types, both in estimated new cases and cancer-related deaths [1, 2]. Depending on the (histological) subtype, up to half of all patients develop metastasis via two major routes of spreading: (i) Delivery of single tumor cells via the bloodstream and/or lymphatic system to the liver or lung; and (ii) locally and directly disseminated single tumor cells, small cell clumps or complete glandulae, that get shed off e.g. during surgery, throughout the peritoneal cavity that induce peritoneal metastasis (PM) [3, 4]. Patients with PM alone or in combination with other metastatic sites, such as the liver and lung, have a dismal prognosis compared to that of patients without PM [5]. Thus, for a long time, PM was regarded as a terminal, 
incurable disease and was mainly treated with palliative chemotherapy and management of complications, such as obstruction, bleeding, or perforation [6].

Two decades ago, peritoneal cytoreductive surgery (CRS) and hyperthermic intraperitoneal chemotherapy (HIPEC) started to improve the survival of patients with PM mostly using mitomycin C (MMC), oxaliplatin, or doxorubicin. However, these therapies are limited to a stringently selected patient cohort and are associated with high morbidity, especially when performed outside of specialized centers [7]. However, data on clinical outcome for all cytoreductive treatments must still be considered controversial, particularly when real-life endpoints are assessed vs. best systemic chemotherapy. Recent studies even propose that CRS alone should be the therapeutic strategy with curative intent for colorectal PM [8]. However, one of the few randomized, prospective trials by Verwaal and others showed an increased median survival from 12.6 to 22.3 months in patients treated with CRS/HIPEC compared to palliative treatment [9]. Another study evaluating the benefit of elevated temperature in rats demonstrated that normothermic intraperitoneal chemotherapy was sufficient to eliminate tumor cells after CRS [10]. Altogether, clinical trials evaluating new treatment options for PM are scarce, and the concept of CRS/ HIPEC invented by Paul Sugarbaker has not changed much over the years, resulting in the use of the same reagents for the last 20 years $[6,11]$.

Current research lacks a suitable human experimental ex vivo model to investigate new therapeutic reagents for HIPEC, as well as the mechanisms underlying PM formation. Existing ex vivo models include inverted bovine urinary bladders used to optimize intraperitoneal drug delivery [12], mouse peritoneum as a scaffold for human tumor cells [13], combined models of human mesothelial and fibroblast cells with rat extracellular collagen matrices [14] as well as mere human models from healthy donors [15-17] or artificial models [18-20]. However, these animal or combined models do not fully reflect the tumor biology including the tumor microenvironment in humans. Thus, new translational research models are urgently needed to investigate and compare alternative treatment options for PM.

Here, we established and characterized a human ex vivo peritoneal coculture model to mimic and investigate PM using either CRC cell lines or patient-derived tumor organoids. Furthermore, this model system was used to analyze possible treatment options for PM, such as HIPEC.

\section{Materials and methods}

\section{Cell culture}

The colorectal cancer cell line HCT116 was obtained from Jens Schmid (Dr. Margarete Fischer-Bosch Institute for Clinical Pharmacology, Stuttgart, Germany). HT29/GFP-luciferase cells were obtained from Julia Beil (University Hospital Tübingen, Eberhard Karls University, Internal Medicine VIII, Tübingen, Germany). Cell lines were authenticated by STR analysis (Eurofins, Ebersberg, Germany). For quality control, mycoplasma PCR using the Venor ${ }^{\circledR}$ GeM Classic kit (11-1025, Minerva Biolabs, Berlin, Germany) was performed according to the manual and before the use of cell lines. HCT116 cells were cultured in RPMI 1640 w/Glutamax (Thermo Fisher Scientific GmbH, Waltham, Massachusetts, US) supplemented with $1 \%$ penicillin/streptomycin (Biochrom AG, Berlin, Germany) and 10\% FCS (Thermo Fisher Scientific $\mathrm{GmbH}$, Waltham, Massachusetts, US) in a $5 \% \mathrm{CO}_{2}$ incubator at $37^{\circ} \mathrm{C}$. HT29/GFP-luciferase cells were cultured in McCoy's 5A Medium w/L-glutamine (Pan Biotech GmbH, Aidenbach, Germany) supplemented with $1 \%$ penicillin/streptomycin and $10 \% \mathrm{FCS}$ in a $5 \% \mathrm{CO}_{2}$ incubator at $37^{\circ} \mathrm{C}$. For coculture experiments 2,500-5,000 cells were seeded onto the tissue. After $24 \mathrm{~h}$ medium replaced with fresh medium to remove cells that did not attach to the tissue.

\section{Organoid culture}

Patient-derived colorectal cancer organoids were generated and cultured as described earlier [21, 22]. In brief, the tissue was cut into small pieces and dissociated at $37^{\circ} \mathrm{C}$. Dissociated cells were passed through a 30 and $100 \mu \mathrm{m}$ cell strainer and collected in advanced DMEM/F12 medium (Thermo Fisher Scientific GmbH, Waltham, Massachusetts, US) supplemented with Glutamax (Thermo Fisher Scientific GmbH, Waltham, Massachusetts, US), penicillin/streptomycin, HEPES, N-acetylcysteine (Merck Chemicals GmbH, Darmstadt, Germany), N-2 supplement (Thermo Fisher Scientific GmbH, Waltham, Massachusetts, US), B-27 supplement (Thermo Fisher Scientific GmbH, Waltham, Massachusetts, US), EGF (PeproTech GmbH, Hamburg, Germany), Y27632 (Absource Diagnostics GmbH, Munich, Germany), and amphotericin (Sigma-Aldrich Chemical, St. Louis, Missouri, US) and embedded in matrigel (Corning B.V., Amsterdam, Netherlands). For subcultivation, organoids were removed from matrigel and dissociated into small organoids using TrypLE (Thermo Fisher Scientific GmbH, Waltham, Massachusetts, US) and then transferred into fresh matrigel. For coculture experiments, organoids were dissociated into small organoids and 2,500-5,000 small organoids were seeded onto the peritoneum.

\section{Peritoneal culture}

Residual tissue from patients with gastrointestinal tumors without peritoneal metastasis undergoing major elective surgery was collected after informed consent. Fresh peritoneal tissue was directly obtained from the surgery room and immediately transferred to the laboratory in E199 medium (Biochrom AG, Berlin, Germany). Afterward, the tissue was incubated for $15 \mathrm{~min}$ in PBS containing penicillin/ 
streptomycin and amphotericin (Sigma-Aldrich Chemie, St. Louis, Missouri, US). Extraperitoneal fat was carefully removed with a scalpel, and small tissue pieces were inserted between two stainless steel rings and cultured with the mesothelial cell surface pointing upward. The tissue was cultured in E199 medium containing penicillin/streptomycin, L-glutamine (Biochrom AG, Berlin, Germany), FCS (Thermo Fisher Scientific GmbH, Waltham, Massachusetts, US), hydrocortisone (Sigma-Aldrich Chemie, St. Louis, Missouri, US), FGF (PeproTech GmbH, Hamburg, Germany), and heparin (Biochrom AG, Berlin, Germany) as described previously [16]. For fresh-frozen sections, the tissue was carefully dislodged from the rings, embedded in Tissue Freezing Medium (Leica Microsystems, Wetzlar, Germany) and immediately frozen in liquid nitrogen. For FFPE sections, the tissue was fixed with 4\% PFA (VWR International, Radnor, Pennsylvania, US) and embedded in paraffin.

\section{Decellularization of peritoneal tissue}

For decellularization, the peritoneal tissue was processed as described before and inserted between two stainless steel rings. Afterward, the tissue was incubated with buffer A (10 mM Tris, $0.1 \%$ EDTA, $\mathrm{pH} 7.8$ ) for $18 \mathrm{~h}$ at $37^{\circ} \mathrm{C}$. The next day, buffer A was removed, and the tissue was washed twice with PBS, followed by incubation with $0.1 \%$ SDS for $24 \mathrm{~h}$ at $37^{\circ} \mathrm{C}$. Thereafter, the tissue was washed three times with buffer B (10 mM Tris, $\mathrm{pH} 7.8$ ) and digested with digestion buffer $(50 \mathrm{U} / \mathrm{mL}$ DNAse, AppliChem GmbH, Darmstadt, Germany, in $20 \mathrm{mM}$ Tris, $2 \mathrm{mM}$ $\mathrm{MgCl}_{2}, \mathrm{pH} 7.8$ ) for $3 \mathrm{~h}$ at $37^{\circ} \mathrm{C}$. Decellularization was confirmed by H\&E staining.

\section{Ex vivo hyperthermic treatment}

Chemotherapeutic drugs were obtained from the in-house clinical pharmacy department, at $2 \mathrm{mg} / \mathrm{mL}$ doxorubicin- $\mathrm{HCl}$ (06581630, Teva GmbH, Ulm, Germany) and $1 \mathrm{mg} / \mathrm{mL}$ MMC (11213532, medac, Wedel, Germany) stock solutions. For HIPEC treatment, the peritoneal tissue was treated with $10 \mu \mathrm{M}$ doxorubicin or $10 \mu \mathrm{M}$ MMC diluted in culture medium for $90 \mathrm{~min}$ at either $37{ }^{\circ} \mathrm{C}$ or $41{ }^{\circ} \mathrm{C}$ in a $5 \% \mathrm{CO}_{2}$ incubator. Afterwards, the tissue was washed gently with PBS and medium containing doxorubicin or MMC was replaced by normal culturing medium. For immunofluorescent evaluation of doxorubicin entry into peritoneal cells, the tissue was embedded immediately after treatment and fresh-frozen tissue sections of $3 \mu \mathrm{m}$ were stained with Vectashield with DAPI (BIOZOL Diagnostica GmbH, Eching, Germany) and analyzed by a Leica TCS SP8 fluorescence microscope. For analysis of cell survival three days after hyperthermal treatment, the peritoneal tissue was digested with $2 \mathrm{mg} / \mathrm{ml}$ collagenase (Sigma-Aldrich Chemie $\mathrm{GmbH}$, St. Louis, Missouri, US) for $24 \mathrm{~h}$ and the luminescent signal of HT29/GFP-luciferase cells was measured using a Bright-Glo ${ }^{\mathrm{TM}}$ Luciferase Assay System (Promega, Madison, Wisconsin, US) according to the manual.

\section{Immunohistochemistry}

FFPE sections of peritoneal tissue $(4 \mu \mathrm{m})$ were stained with Mayer's hematoxylin (Sigma-Aldrich Chemie, St. Louis, Missouri, US) and eosin (Merck Chemicals GmbH, Darmstadt, Germany). After heat-induced epitope retrieval at $\mathrm{pH} 6$ (CD3, CD68), $\mathrm{pH} 9$ (vimentin, CD19, WT1) or proteinase $\mathrm{K}$ treatment (EpCAM), antibody staining (CD3: 1:200,
MRQ-39, Lot-no. 0000052880, Cell Marque, Rocklin, US; CD19: 1:50, MRQ-36, Lot-no. 0000022072, Cell Marque, Rocklin, US; CD68: 1:8000, Kp-1, Lot-no. 1326701B, Cell Marque, Rocklin, US; EpCAM: 1:50, 248M-96, Cell Marque, Rocklin, US; Ki-67: 1:100, clone SP6, monoclonal rabbit IgG, Order-no. 275R-16, LOT-no. 0000075544, Cell Marque, Rocklin, California, US; vimentin: 1:200, V9, Lot-no. 0000036006, Cell Marque, Rocklin, US; WT1: 1:200, 6F-H2, 348M-96, Cell Marque, Rocklin, US) was performed for $25 \mathrm{~min}$ on a Lab Vision Autostainer (Thermo Fisher Scientific GmbH, Waltham, Massachusetts, US).

For extracellular matrix (ECM) staining, the following antibodies and pretreatments were used: Collagen I (ab34710, Abcam, Cambridge, UK, 1:100, no epitope retrieval), collagen III (ab6310, clone FH-7A, Abcam, Cambridge, UK, 1:150, heat-induced epitope retrieval at $\mathrm{pH}$ 6), collagen IV (M0785, Agilent, Santa Clara, CA, US, 1:200, pronase-induced epitope retrieval), MMP9 (NCL-MMP9-439, clone 15W2 Novacastra via Leica Biosystems, Wetzlar, Germany, 1:50, heatinduced epitope retrieval at $\mathrm{pH}$ 9). After antibody-specific epitope retrieval (Pronase $1 \mathrm{~g} / \mathrm{l}, 107433$, Merck, Darmstadt, Germany), endogenous peroxidase blocking (Dako, S2023) was performed for $10 \mathrm{~min}$ at room temperature. Primary antibody staining was performed at $4^{\circ} \mathrm{C}$ overnight, followed by peroxidase/DAB+-based detection using the Dako REAL EnVision Detection System (Dako, K7005). Stainings were evaluated with the help of a trained pathologist from the Department of Pathology at the Robert Bosch Hospital.

\section{Transmission electron microscopy}

Electron microscopy (EM) images were obtained in collaboration with the Institute of Pathology, University Hospital Tübingen, Germany. Tissue samples were fixed with $2.5 \%$ glutaraldehyde (Science Services, Munich, Germany) in cacodylate buffer (Merck, Darmstadt, Germany) at $4{ }^{\circ} \mathrm{C}$ overnight. Thereafter, samples were embedded in araldite (Serva, Heidelberg, Germany), and ultrathin sections were cut using a Leica ultramicrotome (Leica, Wetzlar, Germany). Sections were analyzed in a Zeiss EM-10 transmission electron microscope (Zeiss, Oberkochen, Germany).

\section{Statistics}

Statistical analyses comparing control and treatment conditions were performed using a two-sided Student's t test (paired/unpaired as indicated in the figure legend) in Microsoft Excel 2016. All experiments are shown as the mean and standard error (SE) of at least three independent experiments.

\section{Results}

\section{Establishment of a human ex vivo peritoneal model}

Here, we established and characterized a human ex vivo peritoneal model to investigate PM in a clinically relevant ex vivo system. Human peritoneal tissues were obtained from the surgery room and immediately transferred to the laboratory in sterile tubes. The tissue was washed with 
washing solution and PBS and subsequently inserted between two stainless steel rings for culture, as illustrated in Figure $1 \mathrm{~A}$ and $\mathrm{B}$. For characterization of the tissue and different cell populations within the peritoneum, FFPE blocks and tissue sections were cut before and after culture. H\&E and immunohistochemical (IHC) stainings revealed the integrity of the tissue for up to 25 days of culture. Nucleic structures showed no alteration pointing to no change in viability of the peritoneal tissue (Figure 1C). WT1 positive mesothelial cells were detected for up to 25 days of culture (Figure 1D). In addition, as a sign of viability upon culture, vimentin-positive fibroblasts migrating out from the peritoneum were observed by light microscopy. These fibroblasts could be kept alive and further passaged in culture for up to 25 days (Figure 1E).

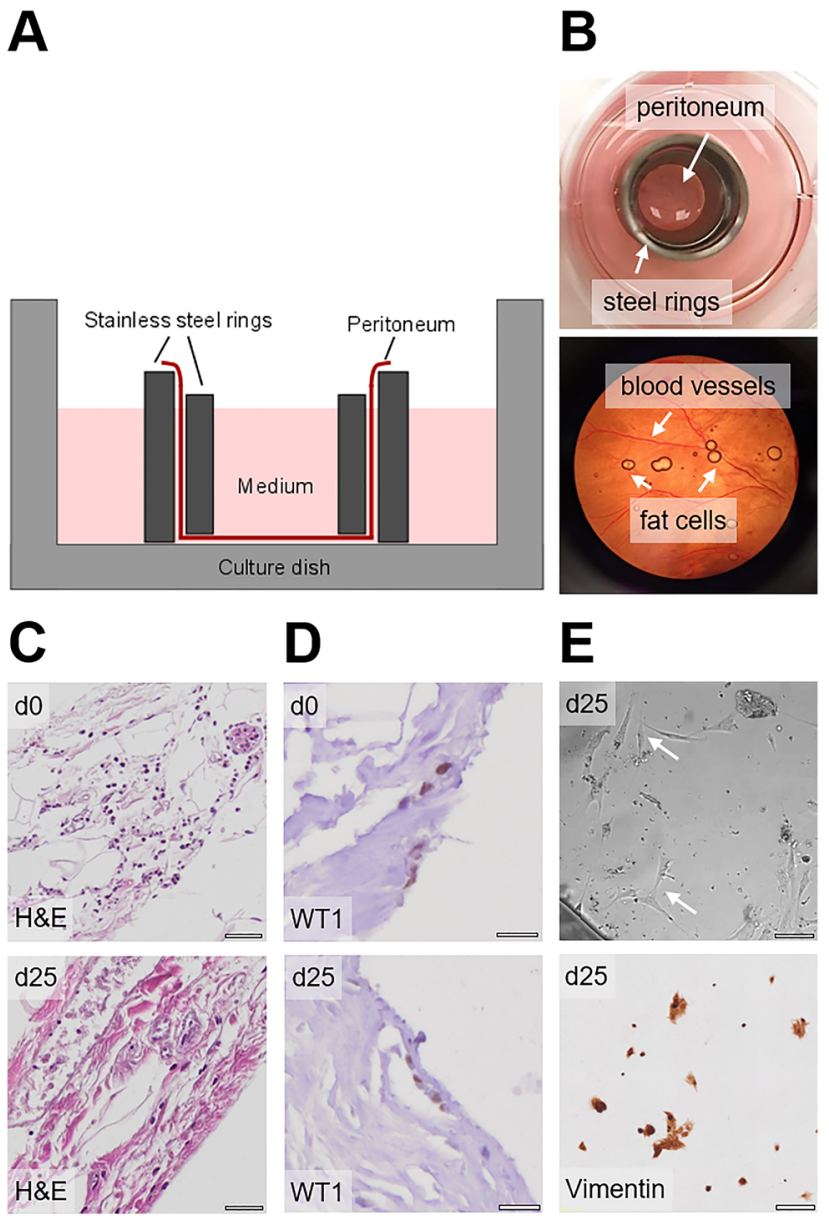

Figure 1: Establishment of a human ex vivo peritoneal model. (A) Schematic setup of the ex vivo peritoneal model. (B) Photographic images of the peritoneal model. (C) H\&E staining of peritoneal tissue on day 0 and day 25 of culture; scale bars $200 \mu \mathrm{m}$. (D) WT1 staining of peritoneal mesothelial cells on day 0 and day 25; scale bars $20 \mu \mathrm{m}$. (E) Light microscopy and vimentin staining of outgrowing fibroblasts on day 25; scale bars $200 \mu \mathrm{m}$.

\section{Immunohistochemical characterization of peritoneal cells and ECM components}

In the next step, we analyzed the different cell populations within the peritoneum. As shown in Figure 2A, vimentin-positive fibroblasts and occasionally CD3positive T-lymphocytes, CD19-positive B-lymphocytes, and CD68-positive macrophages were found in the tissue before culture (d0). Whereas CD3-, CD19- and CD68positive cells could be detected for up to seven days in culture, vimentin-positive fibroblasts were found for up to 25 days. Fibroblasts were widely distributed in the tissue, while immune cells were most frequently found in proximity to vessels. Next, we investigated the distribution of extracellular matrix (ECM) components such as collagens and matrix metalloproteinases (MMPs): We found that collagen I and collagen III were strongly expressed and widely distributed in the peritoneum (Figure 2B), whereas collagen IV was specifically expressed around vessels and in the basal lamina underlying the mesothelial cells. In contrast, the ECM modulator MMP9 was only weakly expressed in the peritoneal cells of donors that did not suffer from PM.

\section{Establishment and characterization of a human ex vivo peritoneal coculture model}

In the next step, we investigated whether peritoneal tissue could serve as an ex vivo model system for PM. As illustrated in Figure 3A, the peritoneal tissue was inserted between two stainless steel rings and cultured with the mesothelial cell surface pointing upward. A sufficient supply with medium to all parts of the tissue was assured at any time during culture. To mimic PM, CRC cell lines were added and cocultured with the peritoneum for up to three weeks. H\&E and IHC staining for the proliferation marker Ki-67 showed that CRC cells attached to and invaded into the peritoneum and could be kept alive for up to 19 days (Figure 3B). To further characterize the peritoneal tissue as well as the coculture model, we performed EM imaging of peritoneal tissue before coculture (Figure 3C, left) and six days after coculture with the CRC cell line HT29 (Figure 3C, middle, right). The mesothelial cell layer was visible before culture (Figure 3C left) and ECM components, such as collagens, were present before culture and in coculture with HT29 cells (Figure 3C left, middle). After six days of coculture, HT29 cells (indicated by white lines) attached to and grew onto the peritoneum (Figure 3C, middle, right). The glycocalyx of HT29 cells was visible as small "bristles". 
A

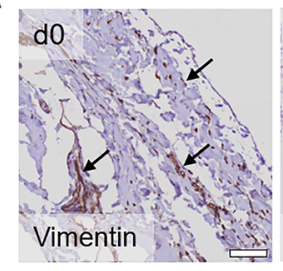

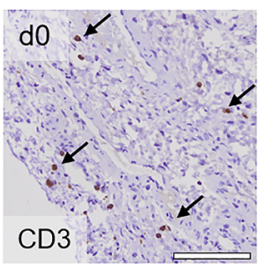
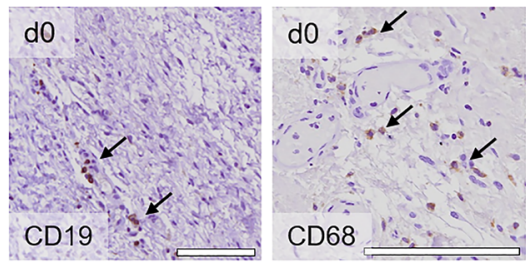

B
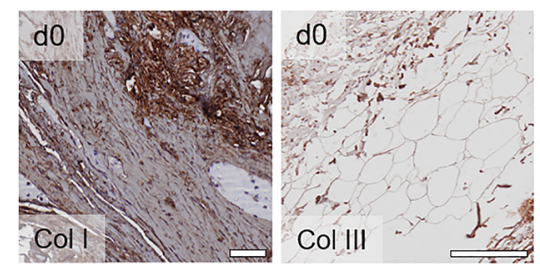

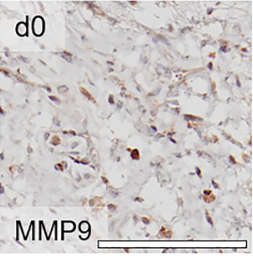

Figure 2: Immunohistochemical characterization of peritoneal cells and ECM components.

(A) Staining for cellular markers before culture (day 0); vimentin: fibroblasts, CD3: T-lymphocytes, CD19: B-lymphocytes, CD68: macrophages; scale bars: $100 \mu \mathrm{m}$. (B) Staining for ECM components before culture (day 0); scale bars $100 \mu \mathrm{m}$.
A

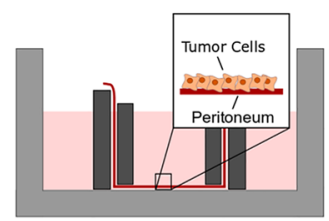

B

C

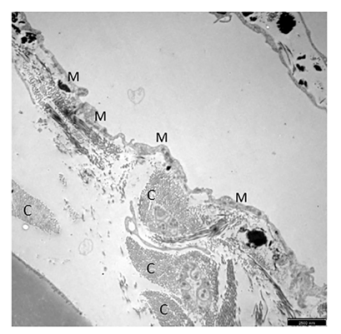

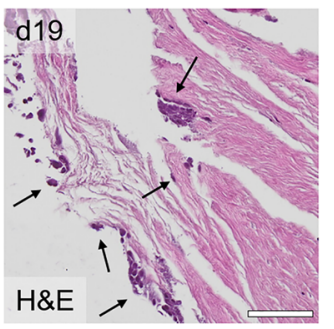

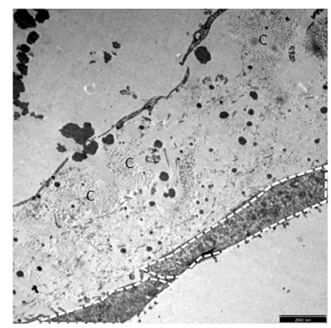

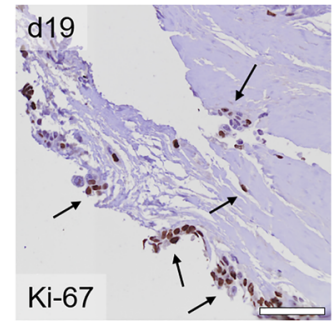

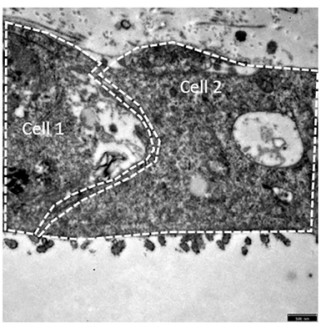

Figure 3: Establishment and characterization of a human ex vivo peritoneal coculture model.

(A) Schematic setup of the ex vivo peritoneal coculture model. (B) H\&E and Ki-67 staining for cocultures with the CRC cell line HCT116 at day 19 after culture; attached and invading CRC cells are indicated by arrows; scale bars $100 \mu \mathrm{m}$. (C) EM images of human peritoneum without (left) and with HT29 cells (middle, right, indicated by white lines) before culture (day 0 , left) and day 6 after coculture (middle, right); $M$ : mesothelial cells, C: collagen, Cell 1 and Cell 2: HT29 cells grown on the peritoneum; scale bars $2500 \mathrm{~nm}$ (left, middle) and $500 \mathrm{~nm}$ (right).

\section{Modeling peritoneal metastasis using patient-derived organoids}

Next, we investigated whether our established ex vivo peritoneal model could serve as a scaffold for patientderived organoids to model PM by CRC tumors. In the course of the disease or during surgery it is possible that small cell clumps or even complete glandulae in addition to single tumor cells get shed off from the primary tumor and metastasize to the peritoneum [4]. For this, patientderived CRC organoids from five different donors were seeded on the peritoneum and cocultured for three days. The growth patterns were analyzed by H\&E and EpCAM staining, showing that organoids attached to and migrated into the peritoneum (Figure 4A and B). To test, whether cell-cell contacts are pivotal for invasion and to investigate the role of ECM components in PM, we cocultured patient- derived organoids on decellularized peritoneal tissue. In this setting, the peritoneal cells were depleted from the tissue leaving only peritoneal ECM components such as collagen fibers. Patient-derived organoids were then seeded onto decellularized peritoneal tissue. After six days of coculture, FFPE sections were cut and stained for ECM components showing that organoids also invaded the peritoneum into deep peritoneal layers of decellularized tissue (Figure 4C). IHC stainings for ECM components revealed a diffuse and widely distributed positive staining for collagen I (Figure 4D), vessels that were positive for collagen I and IV, while organoid margins were only positive for collagen I (Figure 4D and E). Interestingly, organoids were strongly positive for matrix metalloproteinase 9 (MMP9), which is a matrix remodeling enzyme involved in epithelial-mesenchymal transition (EMT) and metastasis formation (Figure 4F). This shows that not only cell-cell 
interactions but also cell-ECM interactions are important for metastasis formation.

\section{Investigating the effects of hyperthermal treatment using a clinically relevant ex vivo peritoneal metastasis model}

PM of colorectal origin can be treated with HIPEC following complete resection of the tumor and peritoneal seeds. However, the clinical benefit of hyperthermia and the exact biological mechanisms are not yet fully understood. Therefore, we investigated whether our established ex vivo peritoneal coculture model could serve as a model system for peritoneal chemotherapeutic treatments such as HIPEC. To determine the influence of temperature on the availability of chemotherapeutic drugs within the peritoneum, we analyzed the percentage of peritoneal cells, which showed an orange fluorescence signal due to intranuclear doxorubicin accumulation following an

A

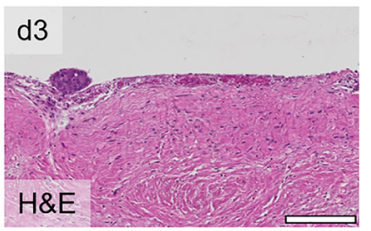

D

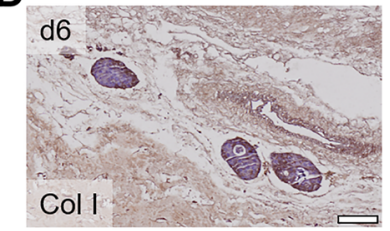

B

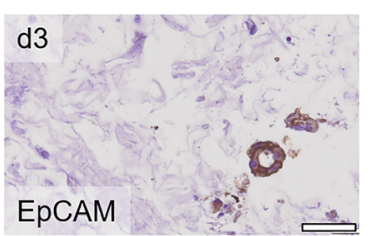

C

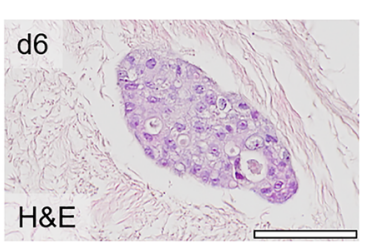

E

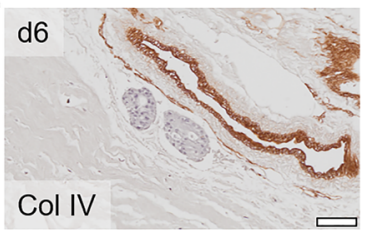

$\mathbf{F}$

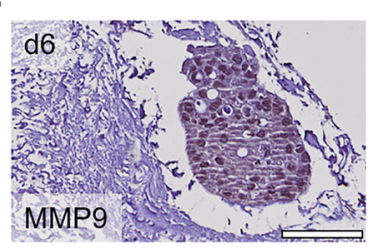

Figure 4: Modeling peritoneal metastasis using patient-derived organoids.

(A) Patient-derived organoids of different donors attached to the peritoneal surface at day 3 after coculture; scale bar $200 \mu \mathrm{m}$. (B) Coculture of patient-derived organoids with cellularized peritoneal tissue after 3 days; sections were stained with EpCAM to distinguish between EpCAM positive tumor cells and negative peritoneal cells; scale bar $40 \mu \mathrm{m}$. (C-E) Coculture of patient-derived organoids with decellularized peritoneal tissue; after 6 days, sections were stained with H\&E (C) and for collagen I (D), collagen IV (E), and MMP9 (F); scale bars $100 \mu \mathrm{m}$.

incubation of the tissue with $10 \mu \mathrm{M}$ doxorubicin for $90 \mathrm{~min}$ at $37^{\circ} \mathrm{C}$ and $41^{\circ} \mathrm{C}$, respectively. Doxorubicin was used in this setting due to its intrinsic fluorescence. As shown in Figure 5B, hyperthermal treatment at $41{ }^{\circ} \mathrm{C}$ significantly enhanced the number of doxorubicin-positive nuclei from 31 to $58 \%(\mathrm{p}=0.007)$.

In further experiments, we examined whether hyperthermal treatment could reduce the survival of cancer cells cocultured with peritoneal tissue. For this, the amount of living cancer cells after treatment was determined by a light signal generated by luciferase-expressing HT29 cells. However, in this set up doxorubicin interfered with the experimental readout due to its intrinsic fluorescence and therefore MMC was used instead. As shown in Figure 5C, hyperthermal treatment for $90 \mathrm{~min}$ at $41^{\circ} \mathrm{C}$ with $10 \mu \mathrm{M}$ MMC significantly reduced the survival of HT29/GFP-luciferase cells within the peritoneum by $23 \%$ compared to treatment at $37{ }^{\circ} \mathrm{C}(\mathrm{p}=0.003)$. Thus, our ex vivo peritoneal model is suitable for modeling and investigating possible treatment options for PM.

\section{Discussion}

In this study, we outlined that a human ex vivo peritoneal coculture model can be used to mimic the PM of CRC cell lines and patient-derived CRC organoids. This model can now be used to investigate current and future treatment options for CRC and PM, such as HIPEC.

The establishment and characterization of a human ex vivo peritoneal model was first shown by Falk et al., who used this model to study mesh-tissue integration in hernia surgery [16]. In this study, we further developed and characterized the peritoneal model, showing that patientderived peritoneal cells could be kept alive in culture for up to 25 days, including the critical mesothelial cells, which was evident by the integrity of the tissue and nucleic structures in H\&E and IHC stainings over time, as well as fibroblasts migrating out from the peritoneum. In addition, we observed that fibroblasts, which had migrated out from the peritoneum, could be kept alive and further passaged in culture for at least two weeks. Moreover, we showed that the human ex vivo peritoneal model is comprised of different cell types, including fibroblasts and immune cells, such as CD3-positive T-lymphocytes. However, additional studies will be needed to further clarify the interaction between these resident immune and cancer cells in our ex vivo model.

Although we noticed some discrepancy to other published ex vivo models using human tissue regarding the viability of the tissue and the integrity of the mesothelial cell layer ex vivo [15], integrity and survival of peritoneal 
A

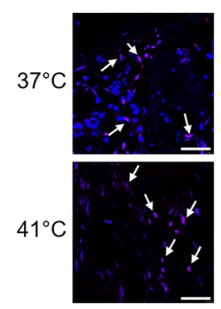

C
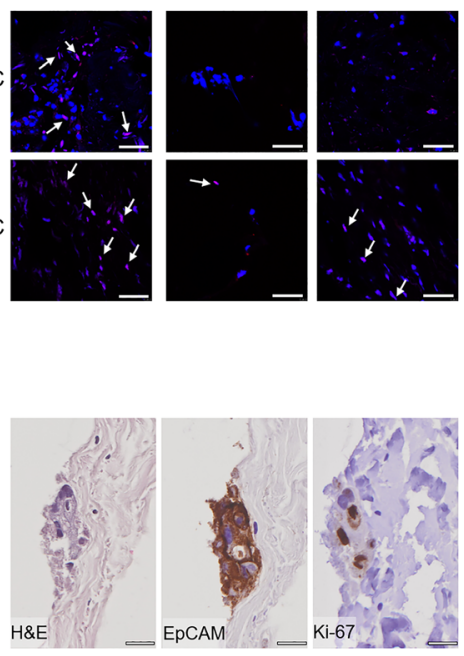

B

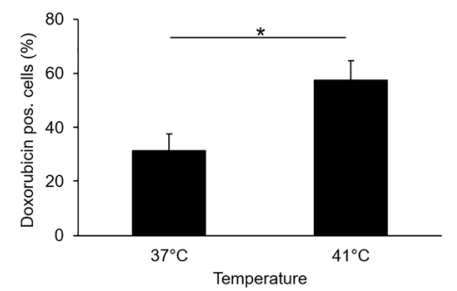

D

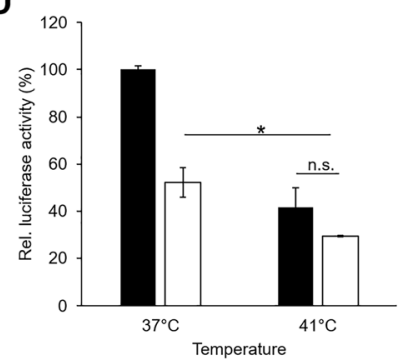

- Ctrl

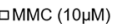

Figure 5: Ex vivo peritoneal model as a model system for hyperthermal chemotherapeutic treatment.

(A) Doxorubicin immunofluorescence (red) of the ex vivo peritoneal model treated with $10 \mu \mathrm{M}$ doxorubicin for 90 min at $37^{\circ} \mathrm{C}$ or $41^{\circ} \mathrm{C}$; doxorubicin (red), nuclei (blue); white arrows indicate doxorubicin-positive cells in three representative images; scale bar, $40 \mu \mathrm{m}$. (B) Percentage of doxorubicin-positive cells following treatment at $37^{\circ} \mathrm{C}$ or $41^{\circ} \mathrm{C}(n=3$; two-sided, paired Student's t test: $p=0.007)$. (C) Coculture of HT29-GFP/luciferase cells with human peritoneum. EpCAM and Ki- 67 stainings on day 6 reveal living and proliferating cancer cells; Scale bar $20 \mu \mathrm{m}$. (D) Treatment with $10 \mu \mathrm{M}$ MMC for $90 \mathrm{~min}$ at $37^{\circ} \mathrm{C}$ or $41^{\circ} \mathrm{C}$ reduces the amount of living HT-29-GFP/luciferase cells 3 days after treatment; shown is one representative of at least three independent experiments performed in triplicates; significance was calculated using a two-sided paired Student's t test; $p=0.003$.

cells during ex vivo culture was comparable to previous publications [16]. Using human residual tissue from different donors results in a batch-to-batch variability and may contribute to the discrepancy seen, but also differences in transport media composition and handling time between tissue removal and arrival in the lab may cause a significant disparity in viability and tissue integration.

In the present model, tumor cells and patient-derived tumor organoids not only attached to but also migrated into the peritoneal tissue. This is an important observation with potential clinical impact, as it shows that tumor cells that are shed off from the primary tumor could attach to and grow into injured sites of the peritoneum, which was already seen in animal models [23-26]. In accordance with that, previous investigations observed a tendency of colorectal tumor cell lines to adhere to traumatized sites of the peritoneum or ex vivo cultured human mesothelial cells [27].

To address the question if and to which extent peritoneal cells and ECM components are involved in PM, we used the ex vivo model in a different set-up: Peritoneal cells were depleted from the tissue and the remaining scaffold consisting of ECM components only was cocultured with patient-derived organoids. We here detected an infiltration of organoids into the tissue indicating that cell-cell interactions between tumor and peritoneal cells might not be the only prerequisite for seeding. In addition, we investigated the distribution of ECM components showing that MMP9, which is involved in matrix remodeling during metastasis, was highly expressed in invading organoids. Similar approaches investigating the influence from the surrounding tumor microenvironment on primary CRC and breast cancer development were recently established using decellularized colon and breast tissue, respectively [28-30]. Therefore, this coculture model is a useful tool to mimic cancer cell attachment and invasion during PM. We here observed a positive immunoreaction for collagen I but not collagen IV around organoids which was uncommon but not unexpected: Although matrigel mainly consists of collagen IV, there seems to be a batchto-batch variability in the composition that was already shown by other groups [31, 32].

Patients with PM have a very poor prognosis [5] and the molecular mechanisms behind PM originating from CRCs are poorly investigated. Moreover, clinically relevant models to investigate PM and possible treatment options apart from animal models are still scarce [15, 17, 33, 34]. Recently Asano and colleagues developed an artificial human peritoneal tissue (AHPT) model to investigate PM of different cancer types [18-20]. This bottom-up approach 
consists of a mesothelial cell layer resting on several layers of human fibroblasts and tubular arranged endothelial cells connected via the cell-accumulation technique [34]. This artificial model provides a reproducible platform for basic research but is time consuming and requires an additional expertise regarding the preparation of the model in advance for any experimental coculture set-up compared to our peritoneal model that is based on residual tissue from human donors. Moreover, by using human residual tissue as basis, we included interindividual differences in tissue architecture, making this model more robust and comparable to the real in human situation. Thus, our established human ex vivo coculture peritoneal model using CRC cell lines and patient-derived CRC organoids helps to investigate the biology of PM and possible treatment options.

HIPEC is a current treatment option for patients with PM that is performed after complete resection of the primary tumor and all visible metastases. However, the molecular mechanisms and benefits of hyperthermal chemotherapy are controversial and not fully investigated $[10,35,36]$. This is, to our knowledge, the first study investigating HIPEC in a human ex vivo peritoneal model. Previous studies by Schaaf et al. already showed that penetration of doxorubicin into the peritoneum was enhanced upon hyperthermia [37]. Here, we used similar concentrations, temperatures, and times for HIPEC treatment of patient-derived peritoneal cells and monitored the entry of doxorubicin into the cells by fluorescence microscopy: Hyperthermia significantly enhanced the entry of doxorubicin into the peritoneum. In addition, hyperthermal treatment with MMC was more effective in killing HT29 cells than MMC treatment at $37{ }^{\circ} \mathrm{C}$ thus showing a clear benefit of higher temperatures for intraoperative chemotherapy. Although the combination of chemotherapy and hyperthermal treatment had a significant impact on cell survival, we did not aim to optimize these treatment conditions in this set of experiments, rather showing the applicability of the model to investigate possible treatment options. Investigating optimal treatment conditions will be part of future studies.

In this study, we established an ex vivo peritoneal coculture model with CRC cell lines or patient-derived organoids that provides a stringent and clinically relevant platform for the investigation of different players during PM and for the elucidation of possible treatment options. Future research based on this model could not only elucidate factors contributing to the formation of PM but also contribute to the development of tailor-made medical and surgical therapies.
Acknowledgments: We thank the Department of Pathology at the Robert-Bosch Hospital for their expert technical help regarding the immunohistochemistry staining.

Research funding: This study was funded by grants DA572(11-4) and RBK-KKF to MHD, and the Robert Bosch Stiftung Stuttgart. The funders had no role in study design, data collection and analysis, decision to publish, or preparation of the manuscript.

Author contributions: Conceptualization: DM, JK, PR, and MHD. Investigation: AM, DM, and JK. Resources: TL, PR, and MHD (tissue), NJ, TM, and MS (organoids), PFB, and WS (EM). Writing - original draft: DM, JK, PR, and MHD. Writing - review \& editing: DM; JK, NJ, TM, WS, MS, TL, PR, HJS, and MHD. All authors have accepted responsibility for the entire content of this manuscript and approved its submission.

Competing interests: Authors state no conflict of interest. Informed consent: Informed consent was obtained from all donors of biological materials included in this study.

Ethical approval: The research related to human use complied with all the relevant national regulations and institutional policies and was in accordance with the tenets of the Helsinki Declaration. This study was approved by the ethics committee of the University Hospital, Tübingen, Germany (696/2016BO2).

\section{References}

1. Sung H, Ferlay J, Siegel RL, Laversanne M, Soerjomataram I, Jemal A, et al. Global cancer statistics 2020: GLOBOCAN estimates of incidence and mortality worldwide for 36 cancers in 185 countries. CA: Cancer J Clin 2021;71:209-49.

2. Siegel RL, Miller KD, Jemal A. Cancer statistics, 2020. CA: Cancer J Clin 2020;70:7-30.

3. Riihimäki M, Hemminki A, Sundquist J, Hemminki K. Patterns of metastasis in colon and rectal cancer. Sci Rep 2016;6:e29765.

4. Pretzsch E, Bösch F, Neumann J, Ganschow P, Bazhin A, Guba M, et al. Mechanisms of metastasis in colorectal cancer and metastatic organotropism: hematogenous versus peritoneal spread. J Oncol 2019;2019:e7407190.

5. Franko J, Shi Q, Meyers JP, Maughan TS, Adams RA, Seymour MT, et al. Prognosis of patients with peritoneal metastatic colorectal cancer given systemic therapy: an analysis of individual patient data from prospective randomised trials from the analysis and research in cancers of the digestive system (ARCAD) database. Lancet Oncol 2016;17:1709-19.

6. Vassos N, Piso P. Metastatic colorectal cancer to the peritoneum: current treatment options. Curr Treat Options Oncol 2018;19:49.

7. Piso P, Dahlke MH, Ghali N, lesalnieks I, Loss M, Popp F, et al. Multimodality treatment of peritoneal carcinomatosis from colorectal cancer: first results of a new German centre for peritoneal surface malignancies. Int J Colorectal Dis 2007;22: 1295-300. 
8. Quénet F, Elias D, Roca L, Goéré D, Ghouti L, Pocard M, et al. Cytoreductive surgery plus hyperthermic intraperitoneal chemotherapy versus cytoreductive surgery alone for colorectal peritoneal metastases (PRODIGE 7): a multicentre, randomised, open-label, phase 3 trial. Lancet Oncol 2021;22: 256-66.

9. Verwaal VJ, van Ruth S, de Bree E, van Sloothen GW, van Tinteren $\mathrm{H}$, Boot $\mathrm{H}$, et al. Randomized trial of cytoreduction and hyperthermic intraperitoneal chemotherapy versus systemic chemotherapy and palliative surgery in patients with peritoneal carcinomatosis of colorectal cancer. J Clin Oncol 2003;21: 3737-43.

10. Klaver YLB, Hendriks T, Lomme RMLM, Rutten HJT, Bleichrodt RP, de Hingh IHJT. Hyperthermia and intraperitoneal chemotherapy for the treatment of peritoneal carcinomatosis: an experimental study. Ann Surg 2011;254:125-30.

11. Sugarbaker PH. Peritonectomy procedures. Ann Surg 1995;221: 29-42.

12. Sautkin I, Solass W, Weinreich F-J, Königsrainer A, Schenk M, Thiel $\mathrm{K}$, et al. A real-time ex vivo model (elBUB) for optimizing intraperitoneal drug delivery as an alternative to living animal models. Pleura Peritoneum 2019;4:e20190017.

13. Asao T, Yazawa S, Kudo S, Takenoshita S, Nagamachi Y. A novel ex vivo method for assaying adhesion of cancer cells to the peritoneum. Canc Lett 1994;78:57-62.

14. Jayne DG, O’Leary R, Gill A, Hick A, Guillou PJ. A three-dimensional in-vitro model for the study of peritoneal tumour metastasis. Clin Exp Metastasis 1999;17:515-23.

15. Cabourne EJ, Roberts G, Goldin R, Ryder T, Mobberly M, Ziprin P. Investigation of tumor-peritoneal interactions in the pathogenesis of peritoneal metastases using a novel ex vivo peritoneal model. J Surg Res 2010;164:e265-272.

16. Falk P, Ruiz-Jasbon F, Strigård K, Gunnarsson U, Ivarsson M-L. An ex vivo model using human peritoneum to explore mesh-tissue integration. Biol Open 2017;6:1391-5.

17. Kenny HA, Krausz T, Yamada SD, Lengyel E. Use of a novel 3D culture model to elucidate the role of mesothelial cells, fibroblasts and extra-cellular matrices on adhesion and invasion of ovarian cancer cells to the omentum. Int J Canc 2007;121:1463-72.

18. Asano Y, Odagiri T, Oikiri H, Matsusaki M, Akashi M, Shimoda H. Construction of artificial human peritoneal tissue by cellaccumulation technique and its application for visualizing morphological dynamics of cancer peritoneal metastasis. Biochem Biophys Res Commun 2017;494:213-9.

19. Odagiri T, Asano Y, Kagiya T, Matsusaki M, Akashi M, Shimoda H, et al. The cell line-dependent diversity in initial morphological dynamics of pancreatic cancer cell peritoneal metastasis visualized by an artificial human peritoneal model. J Surg Res 2021;261:351-60.

20. Oikiri H, Asano Y, Matsusaki M, Akashi M, Shimoda H, Yokoyama Y. Inhibitory effect of carbonyl reductase 1 against peritoneal progression of ovarian cancer: evaluation by ex vivo 3D-human peritoneal model. Mol Biol Rep 2019;46:4685-97.

21. Neef SK, Janssen N, Winter S, Wallisch SK, Hofmann U, Dahlke $\mathrm{MH}$, et al. Metabolic drug response phenotyping in colorectal cancer organoids by LC-QTOF-MS. Metabolites 2020; 10. https://doi.org/10.3390/metabo10120494.
22. Klotz-Noack K, Klinger B, Rivera M, Bublitz N, Uhlitz F, Riemer P, et al. SFPQ depletion Is synthetically lethal with BRAFV600E in colorectal cancer cells. Cell Rep 2020;32:108184.

23. Lejeune FJ. Is surgical trauma prometastatic? Anticancer Res 2012;32:947-51.

24. Zeamari S, Roos E, Stewart FA. Tumour seeding in peritoneal wound sites in relation to growth-factor expression in early granulation tissue. Eur J Cancer 2004;40:1431-40.

25. van den Tol PM, van Rossen EE, van Eijck $\mathrm{CH}$, Bonthuis F, Marquet RL, Jeekel H. Reduction of peritoneal trauma by using nonsurgical gauze leads to less implantation metastasis of spilled tumor cells. Ann Surg 1998;227:242-8.

26. Al Dybiat I, Mirshahi S, Belalou M, Abdelhamid D, Shah S, Ullah $M$, et al. Injured tissues favor cancer cell implantation via fibrin deposits on scar zones. Neoplasia 2020;22:809-19.

27. Falk P, Jonsson A, Swartling T, Ivarsson M-L. Colorectal cancer cells adhere to traumatized peritoneal tissue in clusters, an experimental study. J Invest Surg 2018;31:349-56.

28. Parkinson GT, Salerno S, Ranji P, Håkansson J, Bogestål Y, Wettergren $Y$, et al. Patient-derived scaffolds as a model of colorectal cancer. Cancer Med 2021;10:867-82.

29. Piccoli M, D’Angelo E, Crotti S, Sensi F, Urbani L, Maghin E, et al. Decellularized colorectal cancer matrix as bioactive microenvironment for in vitro 3D cancer research. J Cell Physiol 2018;233:5937-48.

30. Landberg G, Fitzpatrick P, Isakson P, Jonasson E, Karlsson J, Larsson E, et al. Patient-derived scaffolds uncover breast cancer promoting properties of the microenvironment. Biomaterials 2020;235:119705.

31. Hughes CS, Postovit LM, Lajoie GA. Matrigel: a complex protein mixture required for optimal growth of cell culture. Proteomics 2010;10:1886-90.

32. Aisenbrey EA, Murphy WL. Synthetic alternatives to matrigel. Nat Rev Mater 2020;5:539-51.

33. Kenny HA, Dogan S, Zillhardt M, Mitra AK, Yamada SD, Krausz T, et al. Organotypic models of metastasis: a three-dimensional culture mimicking the human peritoneum and omentum for the study of the early steps of ovarian cancer metastasis. Canc Treat Res 2009;149:335-51.

34. Nishiguchi A, Yoshida H, Matsusaki M, Akashi M. Rapid construction of three-dimensional multilayered tissues with endothelial tube networks by the Cell-Accumulation Technique. Adv Mater 2011;23:3506-10.

35. Shimizu T, Murata S, Sonoda H, Mekata E, Ohta H, Takebayashi K, et al. Hyperthermic intraperitoneal chemotherapy with mitomycin $\mathrm{C}$ and 5-fluorouracil in patients at high risk of peritoneal metastasis from colorectal cancer: a preliminary clinical study. Mol Clin Oncol 2014;2:399-404.

36. Wartenberg M, Hescheler J, Acker H, Diedershagen H, Sauer H. Doxorubicin distribution in multicellular prostate cancer spheroids evaluated by confocal laser scanning microscopy and the optical probe technique. Cytometry 1998;31:137-45.

37. Schaaf L, van der Kuip H, Zopf W, Winter S, Münch M, Mürdter TE, et al. A Temperature of $40^{\circ} \mathrm{C}$ appears to be a critical threshold for potentiating cytotoxic chemotherapy in vitro and in peritoneal carcinomatosis patients undergoing HIPEC. Ann Surg Oncol 2015; 22:S758-65. 